\title{
Routing Protocols For Delay Tolerant NeTWORKS: SuRVEY AND PERFormanCE EVALUATION
}

\author{
Luming Wan, Feiyang Liu, Yawen Chen, and Haibo Zhang \\ Department of Computer Science, University of Otago, Dunedin, New Zealand
}

\begin{abstract}
Delay Tolerant Networking (DTN) is a promising technology that aims to provide efficient communication between devices in a network with no guaranteed continuous connectivity. Most existing routing schemes for DTNs exploit the advantage of message replication to achieve high message delivery rate. However, these schemes commonly suffer from large communication overhead due to the lack of efficient mechanisms to control message replication. In this paper we give a brief survey on routing protocols designed for DTNs, and evaluate the performance of several representative routing protocols including Epidemic, Spray and Wait, PRoPHET, and 3R through extensive trace-driven simulations. Another objective of this work is to evaluate the security strength of different routing schemes under common DTN attacks such as the black hole attack. The results and analysis presented in this paper can provide useful guidance on the design and selection of routing protocols for given delay-tolerant applications.
\end{abstract}

\section{KEYWORDS}

Delay Tolerant Network, Routing, Performance Evaluation, Communication Overhead.

\section{INTRODUCTION}

The increasing popularity of ubiquitous computing and communication has led to a huge desire on data exchange between wireless mobile devices, e.g. cell phones, laptops, tablets, and other portable devices, regardless of whether any guaranteed end-to-end connection exists. Delay tolerant networking (DTN) addresses the technical challenges on communication between devices that lose continuous connectivity due to mobility. Currently, DTNs have been applied to a vast of areas, including vehicular networks [1], wildlife tracking [2], and social network analysis [3], etc. However, DTNs are still appearing with numerous of limitations. For example, due to the lack of synchronous end-to-end connectivity, mobile devices have to carry the messages and forward them opportunistically upon encountering the destinations, or forward messages to other relays to help the delivery. This could result in incredible long transmission delay and low message delivery rate. In addition, the constraints on wireless devices, such as storage capacity, communication bandwidth, and battery power, can significantly impact the successful message delivery rate. Hence, routing protocols for DTNs should be able to adapt to the network variation and be efficient to make use of the available hardware resources.

Recent studies exhibit that most of existing routing protocols designed for DTNs appear with high similarity in concepts, but their performances are significantly disparate. Epidemic [4] performs robustly as it blindly floods messages to all devices in the network. Spray and Wait [5] is a flooding-controlled version of Epidemic, as it simply limits the amount of messages that can be 
flooded to the network. In most prediction-based schemes, such as PRoPHET [6], MaxProp [7] and PER [8], the message forwarding decisions are made based on a quality metric called encounter predictability which is accumulated from past encounters. However, the above prediction-based schemes ignore some important encounter information, such as the contact time and contact duration. Some studies demonstrate that human activities appear with high repetition, such as weekly meetings. The performance of routing schemes could be enhanced significantly through the exploitation and utilization of those regular patterns. 3R [9] is a fine-grained historybased routing scheme, by which the contact time of pair-wise encounters are perfectly recorded. Due to the maintenance of fine-grained encounter history, the encounter predictability in $3 \mathrm{R}$ is time-dependent, that is, it can be calculated based on only the past contacts that occurred in the same period as the lifetime of the packet, rather than making a long-term average estimation as in most prediction-based protocols such as PRoPHET. In addition, 3R is a forwarding-based scheme. Each node always forwards the original message to the next good relay rather than transmitting replicas as in all the protocols mentioned above. However, single-copy for each message is not ideal for DTNs since message could be easily lost due to unstable connectivity, message buffer overflow, and other uncertainties.

It is difficult to design an absolutely perfect routing protocol that suits to all DTN applications. The performance of a routing protocol can be affected by a large number of factors, such as the popularity and active rate of nodes, different setting of parameters for mobile devices, etc. In this paper, we evaluate the performance of several most popular routing protocols, including Epidemic, Spray and Wait, PRoPHET, and 3R through trace-driven simulations. Also, we investigate the impact of different parameters of Spray and Wait and PRoPHET to achieve their best performance. The aim of this work is to provide some important guidance on routing protocol design and selection for delay tolerant networks.

On the other hand, it is challenging to develop a secure routing scheme for DTNs due to the vast constraints such as hardware limitation and unstable end-to-end connectivity. Oversimplified routing protocol are highly vulnerable to be attacked. Byzantine attacks[14] summarized several typical categories of attacks in DTNs. Most existing routing protocol for DTN cannot address the Byzantine attacks. In this paper, we also evaluate the security strength of several protocols with the black hole attack, which is a representative class of Byzantine attacks.

The reminder of this paper is organized as follows: Section 2 introduces the protocols we are going to evaluate in detail. Section 3 introduces the data traces and experiment setup. Section 4 shows the performance evaluation in two perspectives. Firstly, we present the impact of different parameter settings for Spray and Wait, and PRoPHET protocols. Then, we evaluate the performance of all the protocols with their optimal parameter settings. This includes the general performance comparison, the average message delivery latency of each protocol, and the performance difference of the protocols under various packet longevity. In Section 5, we measure the security strength of the protocols. The paper is concluded in Section 6.

\section{ROUTING PROTOCOLS}

This section introduces the details of four protocols, Epidemic, PRoPHET, Spray and Wait, and $3 \mathrm{R}$, that will be evaluated in our simulations. 


\subsection{Epidemic}

Epidemic [4] is a pure flooding-based routing protocol for DTNs. Each device (source or relaying node) always propagates message replicas to all of the contactable nodes until the message is received by the destination node or the message deadline expires. Due to the nature of unlimited flooding, Epidemic is able to achieve the best message delivery rate when each node has an infinite memory buffer, but its performance deteriorates significantly when each node only has limited resources. This is because numerous of message replicas have to be dumped due to memory overflow.

\subsection{Spray and Wait}

Similar to Epidemic, Spray and Wait [5] is also a flooding-based routing protocol, but it controls the message flooding by directly limiting the number of replicas that an original message can produce and forward. In spray phase, each message can only propagate a constant number $L$ of replicas. The source node forwards only one replica to the next node it encounters. The source node keeps propagating replicas until there is only one left, then the source node and all relays it encountered before switch to the wait phase. That is, those messages only wait for the destination node encountering their holders rather than further spreading replicas to other relays.

There is a derivation version of Spray and Wait called Binary Spray and Wait. Instead of perform pure flooding like Spray and Wait, Binary Spray and Wait propagate its message copies in a binary tree manner. Suppose a message is allowed to spread $L$ replicas, the source node will generate $L$ replicas in the beginning and spread them among encountered relays. Suppose a node $A$ has $n(1 \leq n \leq L)$ replicas of message $m$, and it encounters a node $B$ with no replica of $m$ previously. $A$ will send $\left\lceil\frac{n}{2}\right\rceil$ replicas to $B$ and keep the rest of $\left\lfloor\frac{n}{2}\right\rfloor$ replicas to wait for more relaying nodes. When only one replica is left in $A$ or any other relaying node, they stop spreading any replica and wait for encountering the destination node. Spray and Wait can achieve a tradeoff between the delivery rate and transmission overhead by setting a proper limitation of $L$.

\subsection{PRoPHET}

PRoPHET [10] is a prediction-based scheme, and it is one of the few DTN routing protocols that have an IETF draft. It implements a quality metric called encounter predictability to measure the capability of the encountering nodes whether or not it can transmit the message to the destination. A replica of a message is propagated to the encountering node if it has a higher predictability than its holder. This guarantees that each time a message can always be propagated to a better relay. The encounter predictability is integrated in three perspectives, which are direct probability, transitivity and ageing. Direct probability is the probability of transmitting a message directly between two nodes. It is updated whenever two nodes directly encounter each other as follows:

$$
P(a, b)=P_{\text {old }}(a, b)+\left(1-\delta-P_{\text {old }}(a, b)\right) \times P_{\text {encounter }},
$$

where $P_{\text {old }}(a, b)$ is the encounter probability of node $a$ and $b$ before the current encounter occurs, $P_{\text {encounter }} \in[0,1]$ is a scaling factor at which the probability increases on encounters, and $\delta$ is a small positive value to set an upper bound on $P(a, b)$.

Transitivity estimates the probability of indirect contact that through multiple-hop relays, as shown below: 


$$
P(a, c)=P_{o l d}(a, c)+\left(1-P_{o l d}(a, c)\right) \times P(a, b) \times P(b, c) \times \beta,
$$

where $P(a, c)$ is the encounter probability between node $a$ and $c$ through relay node $b, P_{\text {old }}(a, c)$ is the probability from previous time of update, and $\beta \in[0,1]$ is a scaling factor that decides how large the impact of transitivity should have on the encounter predictability.

PRoPHET uses an ageing mechanism to decay the encounter predictability and eliminate the long-time inactive nodes, as shown below:

$$
P(a, b)=P_{\text {old }}(a, b) \times \gamma^{k},
$$

where $\gamma \in[0,1]$ is the ageing constant, and $K$ is the number of time units elapsed since the last time $P(a, b)$ was aged.

As shown in the above Equations, there are three important parameters for PRoPHET protocol: scaling factors for direct delivery $P_{\text {encounter }}$, transitivity $\beta$, and the ageing constant $\gamma$. They have significant influence on the performance and overheads of PRoPHET scheme. PRoPHET can be an efficient routing protocol by setting the proper parameters if the encountering patterns in DTNs are predictable, because the messages and replicas are only forwarded to the relays with more benefits on message delivering. At present, there are two versions of PRoPHET protocol: PRoPHET'03 [6] and PRoPHET'12 [10]. They have different recommendation settings for these parameters, while the principle of message forwarding for both versions are basically identical. PRoPHET'12 [10] has slight improvement on its routing mechanism by filtering out the transient contacts. These transient contacts are not long enough for nodes to exchange messages, and thus they should not be counted into history and take effect to the predictability.

\subsection{R}

$3 R$ [9] characterizes the encounter history in a fine-grained form by storing more information for each encounter, including the nodes that the encounter occurs, the start time and the end time of each encounter. Each node maintains a table for the encounter information of its contacted nodes. The fine-grained history in a node will group the contacts according to different types of the day \{weekday, weekend\}. Each item in the table represents a fixed time interval of the real world (e.g. 1 hour), and it records the overall contact frequency between the table holder and the specified node at exact the same period in the past. Suppose a message $m$ has a lifetime that spans $k$ slots. Let $F_{i}^{a}$ be the number of contacts of node $a$ that occurred at a time slot $i$ in the past, and $F_{a b}^{i}$ be the number of contacts occurred between node $a$ and $b$ at time slot $i$, the estimated contact probability $\mathrm{p}_{\mathrm{ab}}^{\mathrm{i}}$ at slot $i$ is:

$$
p_{a b}^{i}=\frac{F_{a b}^{i}}{F_{a}^{i}} .
$$

Suppose the lifetime of a message $m$ spans $k$ time slots, the overall probability that message $m$ can be delivered from node $a$ to node $b$ before it expires is estimated by integrating the probabilities of these $k$ slots as follows:

$$
P_{a b}(k)=1-\prod_{i=0}^{k}\left(1-p_{a b}^{i}\right) .
$$

Due to the maintenance of fine-grained history, a time-dependent forwarding prediction is enabled by only figuring out the delivery probability within the lifetime of a message, rather than 
calculating a long-term average estimation. Similar to PRoPHET, 3R also compares the predictability and forwards messages to nodes with higher delivery probability. Whereas, $3 \mathrm{R}$ only directly forwards the original message to the next relay, instead of sending a replica as in PRoPHET. Due to the predictability calculation based on fine-grained contact history and singlecopy message forwarding, 3R can effectively reduce delivery overheads with no much loss on message delivery rate.

\section{Data Traces and Experiment Setup 3.1. Data Traces}

We evaluate the above four representative routing protocols for DTNs with extensive trace-driven simulations. To increase the accuracy and reliability of our evaluation, we use two realistic DTN data traces: INFOCOM [11] and MIT Reality [12], which are obtained from the open-source website CRAWDAD [13].

- INFOCOM: This data trace consists of Bluetooth sightings and it records 4 days of contacts occurred during the INFOCOM'05 conference. In the experiment, 24 internal devices monitored the network and more than 200 external devices were discovered. Each device has a scan granularity of 120 seconds, and each scan lasted for 5 seconds.

- MIT Reality: This trace contains the contact information including communication, proximity, and location from faculties and students at MIT over the course of the 2004-2005 academic year. To collect this trace, 89 devices were used to record the contacts, and more than 20,000 devices were discovered. Each device scans to discover neighbors every 5 minutes. In our simulations, we use the devicespan subtrace that records Bluetooth contacts for 1 month.

\subsection{Experiment Setup}

In our simulations, the routing schemes are evaluated using the same message trace and contact trace. To avoid the negligible delivery rate caused by numerous long-time inactive nodes which actually seldom communicate, we randomly select the source node and the destination node of a message from 20 most active nodes during the lifetime of this message. In the INFOCOM trace, each source generates a message with the probability of 0.15 in every 600 seconds, whereas the probability is 0.6 in the MIT Reality trace. The lifetime of each message varies from 2 hours to 3 days if not specially notified. The size of each generated message is randomized from $2 \mathrm{k}$ bytes to 100k bytes.

For the MIT Reality trace, the network is warmed up for one week at the beginning of each simulation run. This avoids the inaccurate prediction on encounter probability for predictionbased protocols, i.e. PRoPHET and 3R, when the network is just booted up. Also, we reserve 3 days at the end of simulations to avoid any message left in the network after the simulation is completed. During these warm-up and sinking periods, messages are not allowed to be generated. This process is not available for the INFOCOM data trace, since it only contains 4 days of contact traces.

\section{Performance Evaluation}

In this section, we firstly show the performance of Spray and Wait and PRoPHET with different parameter settings using the INFOCOM data trace, since they have some specific parameters. 
Then we investigate the performance of all four protocols, i.e. Epidemic, Spray and Wait, PRoPHET, and 3R, through trace-driven simulations. The performance of these four protocols are investigated in three perspectives: different memory buffer sizes, average message delivery latency, and packet lifetimes. The performance is evaluated using the following three metrics:

- average message delivery rate: the proportion between the number of successfully delivered messages against the number of original messages.

- message overhead $(M O)$ : the ratio of the number of replicas against the number of original messages.

- communication overhead $(\mathrm{CO})$ : the ratio of the difference between the number of delivered messages and hop-by-hop transmissions to the number of delivered messages.

The parameter setting for Spray and Wait is slightly different in each data trace in accordance with the characteristic of the data trace. The Binary Spray and Wait protocol is implemented in the entire experiments as it performs slightly better than its original version. Due to the huge difference on popularity of nodes in these two traces, the maximum number of replicas for Binary Spray and Wait with INFOCOM trace is set to 5, and 70 with MIT Reality trace. The parameters in PRoPHET are configured with the values provided in PRoPHET'12 [10] by default. All the nodes are set with infinite memory buffer if not specified. All the protocols dump messages using the First In First Out policy when the message buffer is full, that is, the first message in the buffer queue (oldest) is dumped to prevent buffer overflow.

\subsection{Impact of Parameters for Spray-and-Wait and PRoPHET}

Spray-and-Wait: Fig. 1 shows the performance of the original and binary version of Spray and Wait routing protocol with different maximum number of replicas. In Fig. 1(a), it can be seen that the original Spray and Wait achieves higher delivery rate than the binary version when the maximum number of replicas is less than 10 . With the further increase on the number of replicas, the binary version performs better than the original version. That is because the original scheme can only spread the replicas around the neighbours, while the binary scheme can spread the replicas more widely in a binary-tree manner if enough number of replicas are allowed. We can see the delivery rate of both protocols remain steady when the maximum replicas exceeds 40 .

The highest average delivery rate achieved by the original and the binary versions are 0.9314 and 0.9382 , respectively. That means the number of replicas are enough for two Spray and Wait protocols with no further benefits for more replicas. Meanwhile, MO and $\mathrm{CO}$ for both schemes appear with the similar increasing trend as shown in Fig. 1(b) and (c). The MO and CO of the original Spray and Wait increase apparently with the maximum replicas less than 40, and they stay constant afterwards also due to the limitation on the number of neighbours, whereas both two kinds of overheads of Binary Spray and Wait grow almost linearly since the replicas can be spread to more relay nodes on the network. From these figures, it can be seen that with a large number of replicas, the multi-hop routing of Binary Spray and Wait is able to perform better than the only two-hop routing in original version, but it suffers almost twice of overheads than the original version. 

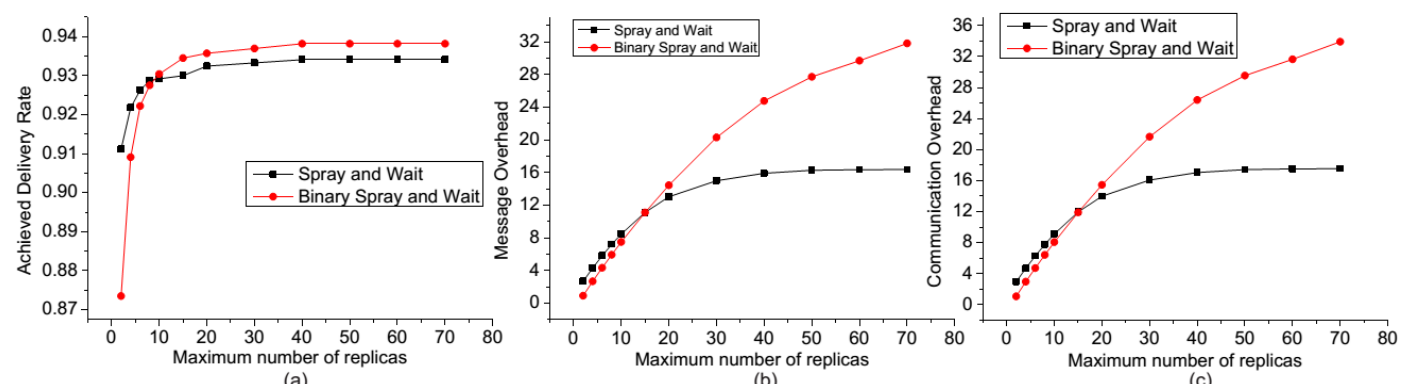

(a)

Fig. 1. The performance of Spray and Wait with different number of message replicas, (a) delivery rate, (b) message overhead, (c) communication overhead.

PRoPHET: The performance of PRoPHET routing protocol is critically impacted by the parameters shown in Table 1. PRoPHET provides totally different recommended parameters in their 2012 version [10] compared to the 2003 version [6].

Table 1. PROPHET recommended parameters

\begin{tabular}{|c||c|c|c|}
\hline Parameters & $\mathrm{P}_{\text {encounter }}$ & $\mathrm{B}$ & $\Gamma$ \\
\hline \hline PRoPHET 2003 & 0.75 & 0.25 & 0.98 \\
\hline PRoPHET 2010 & 0.5 & 0.9 & 0.999 \\
\hline
\end{tabular}

Fig. 2 shows the performance of PRoPHET'03 and PROPHET'12.

In these experiments, each node has infinite memory buffer to store messages and replicas. The principle of message forwarding for these two versions are basically identical. It can be seen that in Fig. 2(a), the delivery rate is increased by $0.5 \%$ with the parameters of PRoPHET'12 in comparison with PRoPHET'03. A larger ageing parameter $\gamma$ makes PROPHET'12 better to tolerate the variation of contact patterns and achieve higher delivery rate. However, both MO and $\mathrm{CO}$ are raised by $6.1 \%$ and $5.4 \%$ respectively as shown in Fig. 2(b). This is because a larger $\beta$ in PRoPHET'12 leads to higher impact on the transitive connectivity, and a lower $\mathrm{P}_{\text {encounter }}$ reduces the impact of direct delivery. Hence, more replicas need to be generated and forwarded by the relay nodes.

\subsection{Performance Evaluation with Different Memory Sizes}

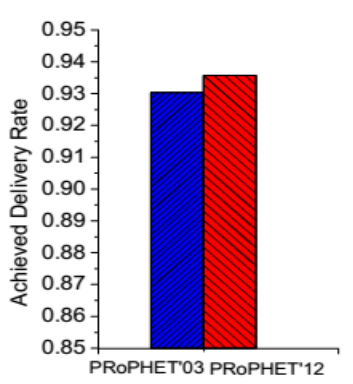

(a)

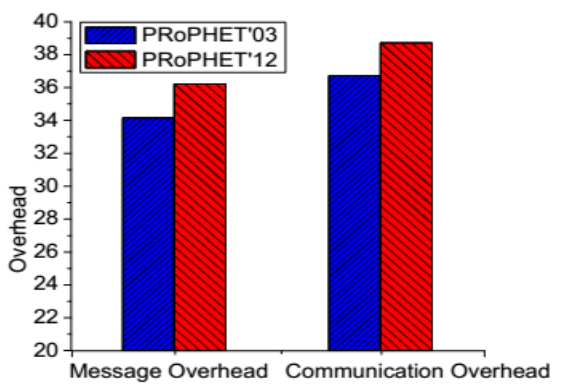

(b)

Fig. 2. PRoPHET'03 versus PRoPHET'12, (a) delivery rate, (b) overheads. 
In each mobile device, the memory buffer used for message delivery always has a limited size. The available memory has huge influence on the delivery rate and overheads. We evaluate the performance of four routing protocols with different memory sizes using INFOCOM and MIT Reality traces.

(1) INFOCOM Trace: In Fig. 3(a), we evaluate the influence of memory size on the delivery rate for different routings. It can be seen that the delivery rates of all four routing protocols have a sharp increasing trend with a small memory buffer $(<10 \mathrm{MB})$, and then they tend to remain stable even if the memory buffer size keeps growing. Binary Spray and Wait has more outstanding performance than other three routings when each node has a memory buffer of $80 \mathrm{M}$ bytes or less. It reaches its maximum delivery rate of 0.923 with the memory buffer size increasing to $60 \mathrm{M}$ bytes, and the delivery rate tends to be stable afterwards. That is because it is a controlled flooding scheme ( 5 replicas) and has less message dropped due to memory overflow than other routings. When the memory size exceeds $60 \mathrm{MB}$, there will be no message dumps for this routing and the delivery rate will be stable. Similarly, 3R also has better performance with a smaller memory buffer size compared with Epidemic and PRoPHET. It has less requirement on memory size because of single-message and its limitation on message forwarding. However, it reaches the maximum delivery rate of 0.792 at the memory size of $40 \mathrm{M}$ bytes, which is much smaller compared with the other three protocols. That is because 3R only forwards the original message. Although the delivery rates of Epidemic and PRoPHET raise slowly, their maximum delivery rates are higher than $3 \mathrm{R}$ and Spray and Wait (0.941 and 0.930 respectively) since they are memory-hungry schemes. Epidemic protocol needs large amounts of memory for unlimited flooding and can achieve the highest delivery rate with enough memory. PRoPHET also needs a large memory size for transmitting the amounts of messages and replicas.

Fig. 3(b) and (c) evaluate the overheads with different memory sizes. We can see that Epidemic always has the highest MO and CO due to the nature of unlimited flooding. Since it needs to generate more replicas when encountering relay nodes with high predictability, PRoPHET also has higher overheads, which is almost half of the overheads as Epidemic. Because the maximum number of replicas in Binary Spray and Wait is set to 5, the maximum MO and CO of Binary Spray and Wait scheme tend to be 5 even with a much larger memory size. Since $3 \mathrm{R}$ is a singlemessage forwarding-only scheme, there is no message overhead. The $\mathrm{CO}$ of $3 \mathrm{R}$ is also lowest because the message is only forwarded to a node with higher predictability according to finegrained encounter history. The CO of Binary Spray and Wait and 3R appears with a decreasing trend as the memory size increases, since they have limitations on message forwarding, and a larger memory size increases the number of delivered messages by reducing message dropping.
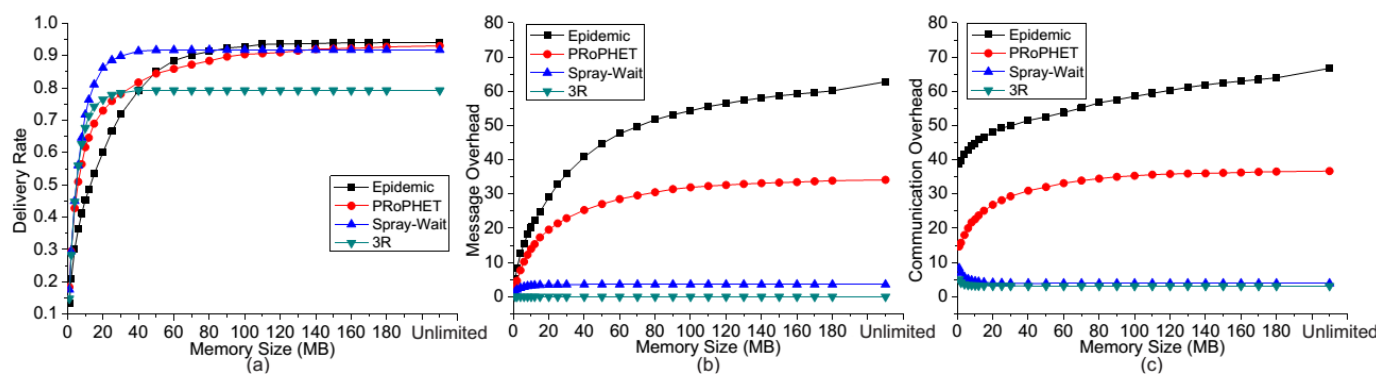

Fig. 3. Performance evaluation with different memory sizes using INFOCOM trace, (a) delivery rate, (b) message overhead, (c) communication overhead. 


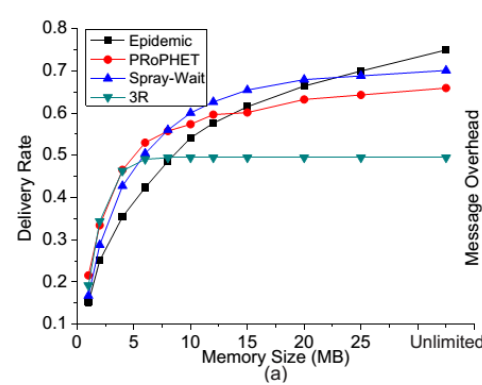

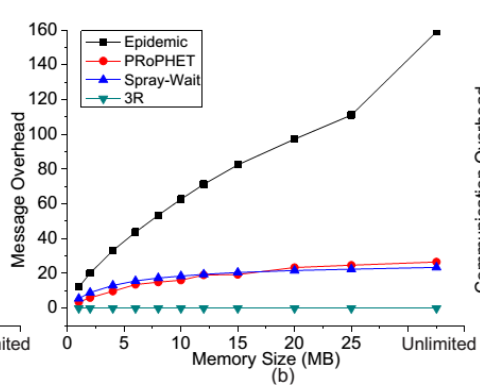

(b)

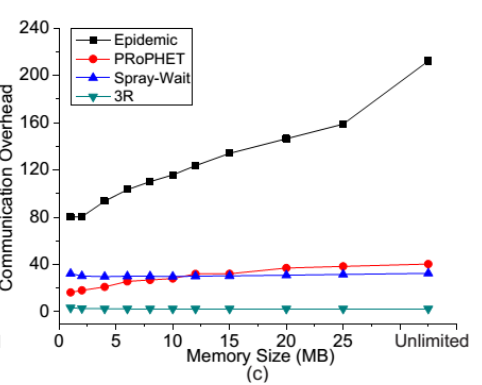

(c)

Fig. 4. Performance evaluation with different memory sizes using MIT Reality trace, (a) delivery rate, (b) message overhead, (c) communication overhead.

(2) MIT Reality Trace: As shown in Fig. 4(a), the delivery rate of each routing protocol has similar trend as the memory size increases. However, PRoPHET and 3R have better performance than Epidemic and Spray and Wait when the memory size is small $(<5 \mathrm{MB})$. Since the MIT Reality trace lasts for 1 month and these two routings can make use of the contact history to get more accurate prediction for message forwarding, and a smaller memory is relatively enough due to less replicas and message forwarding. As the memory size increases, Epidemic and Spray and Wait achieve higher delivery rate because a large number of flooding replicas can be tolerated in the memory with few of message dumps.

Similarly, the message overhead (MO) in Fig. 4(b) and communication overhead in Fig. 4(c) vary in the same way as the memory size increases. Since there are more nodes in the MIT Reality data trace than that in the INFOCOM trace, the overheads of Epidemic are significantly increased. That means Epidemic is not suitable for DTNs with a large number of devices. We can see that the overheads of Spray and Wait increase with the increase of maximum allowed replicas (from 5 in INFOCOM trace to 70 in MIT Reality trace). PRoPHET and 3R protocols have lower overhead for both data traces, which is determined by the popularity of nodes and the activeness of each node.

From this set of simulations, it can be seen that flooding-based routing protocols can achieve better delivery rate, but require much larger message memory. Whereas, the prediction-based routing protocols are more memory efficient, and can achieve desirable delivery rate with very low overheads if they have accurate prediction on the contact patterns.

\subsection{Average Latency}

We also conduct a set of simulations to measure the routing efficiency of each DTN protocol in term of the average message delivery delay. The message delivery latency is defined as the time span from the time the message is generated until the time when the original message or one of its copy reaches the destination. We evaluate the average message delivery latency of the four routing schemes using the INFOCOM and MIT data traces separately with different memory capacities. 


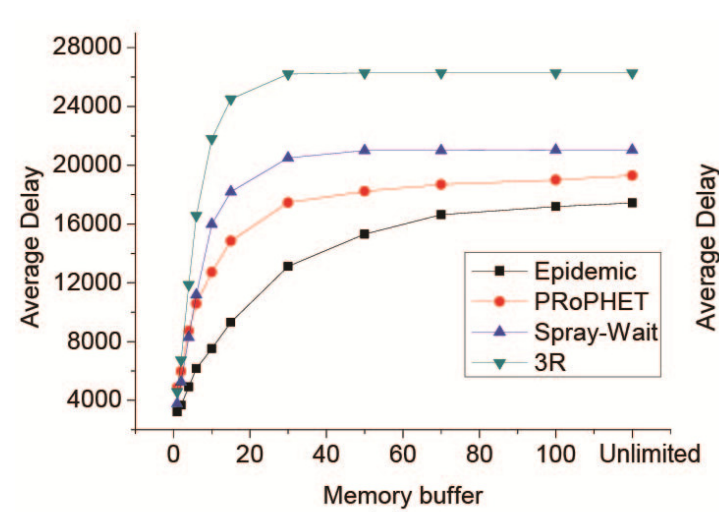

(a)

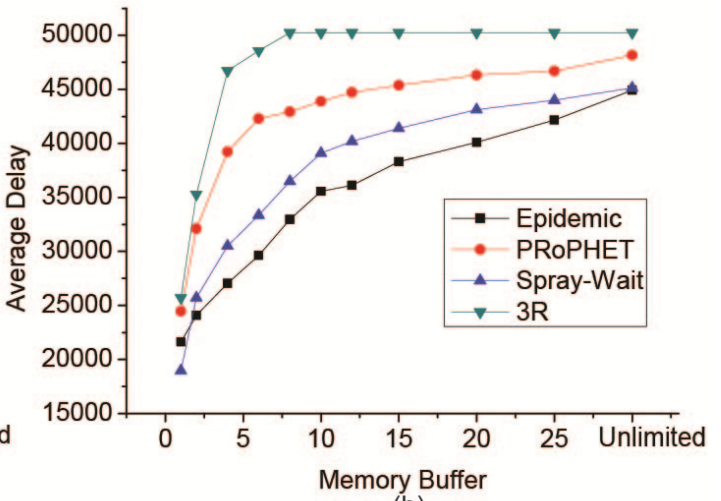

(b)

Fig. 5. Average message delivery latency with different memory capacity using trace (a) INFOCOM, (b)

$$
\text { MIT Reality }
$$

(1)INFOCOM trace: In Fig.5 (a), all of the protocols appear with a similar increasing trend. Epidemic spends the least average time on message delivery for all cases studied because it takes the advantage of blind flooding. Under the situation of a large memory buffer at each node, Epidemic has the shortest delay because messages can survive longer at nodes. Large number of message copies of a single message produce a higher chance for the message to reach its destination through opportunistic encountering between nodes, rather than waiting for frequent contact nodes like PRoPHET and 3R. On the other hand, when nodes have very limited memory buffer, most of the message copies have to be discarded because of memory overflow. Thus the delivery rate is incredible low for Epidemic. However, these limited amount of deliverable messages still takes the benefit of flooding. The messages can be flooded to the destination through a relative short path, so that Epidemic still has the shortest delay with the extremely limited memory at nodes. PRoPHET costs slightly more than Epidemic. It takes the second place because of its accurate routing prediction. The performance of $3 R$ is relatively poor because of its single-message-forward mechanism. The use of only a single message is hard to find out the shortest path to the destination in DTN.

(2)MIT Reality trace: Similar to Fig.5 (a), Epidemic and 3R still get the first and the last place on the average message delivery delay. However, Spray and Wait outperforms PRoPHET in MIT Reality trace. This is because this trace contains a much larger node population. The upper limit of message copy is set to 70, which is a huge increase against 5 in INFOCOM trace. Therefore, Spray and Wait performs almost the same degree of flooding as Epidemic, so that messages can be rapidly delivered to their destination through such flooding.

From the results above, flooding-based routing protocol always gain a lower latency than prediction-based all the time. However, combining the result in Fig. 3 and Fig. 4, flooding-based protocols have a poor performance when nodes have limited buffer. In contrast, flooding-based protocols obtains incredible high overheads with large memory buffer. It is necessary to comprehensively inspect the routing protocols to make a fair comparison, rather than only observe a single aspect.

\subsection{Performance Evaluation with Different Packet Lifetimes}

Each message may experience an unpredictable delivery latency in DTNs due to the lack of guaranteed continuous end-to-end connectivity. Therefore, in order to achieve more effective communication for DTNs, each message needs a suitable lifetime either by users setting or 
default configuration of specific devices. In this set of simulations, we evaluate the influence of different packet lifetimes for the routing algorithms using INFOCOM and MIT Reality data traces respectively. We assume each node has an infinite buffer size.

(1) INFOCOM Trace: Fig. 6 shows the delivery rate and overheads of four routing protocols with different setting of packet lifetimes using the INFOCOM trace. In Fig. 6(a), it can be seen that for each routing protocol the delivery rate appears with a dramatic increase when the packet lifetime increases from 6 to 30 hours. For example, the delivery rate increases from 0.454 to 0.832 for $3 \mathrm{R}$ routing. The reason is that the probability of directly encountering the destination or the message delivered to the destination by multi-hop forwarding grows when the packet lifetime increases. This increasing trend slows down when the packet lifetime exceeds 30 hours. That is because the data trace only lasts 4 days, and the contact pattern is short-time dependent. The delivery rate will not increase even with a large packet lifetime since the destination node may only be active in a short time period. The overheads shown in Fig. 6(b) and (c) also demonstrate that, for Epidemic and PRoPHET, the overheads increase as the packet lifetime increases, since more replicas can be generated and forwarded to the destination. Whereas, the lifetime is enough long for message delivery when it exceeds 30 hours in this trace. The overheads of Spray and Wait and 3R keep stable with different packet lifetime, because the number of replicas is limited in Spray and Wait and 3R is a single-message forwarding based scheme.
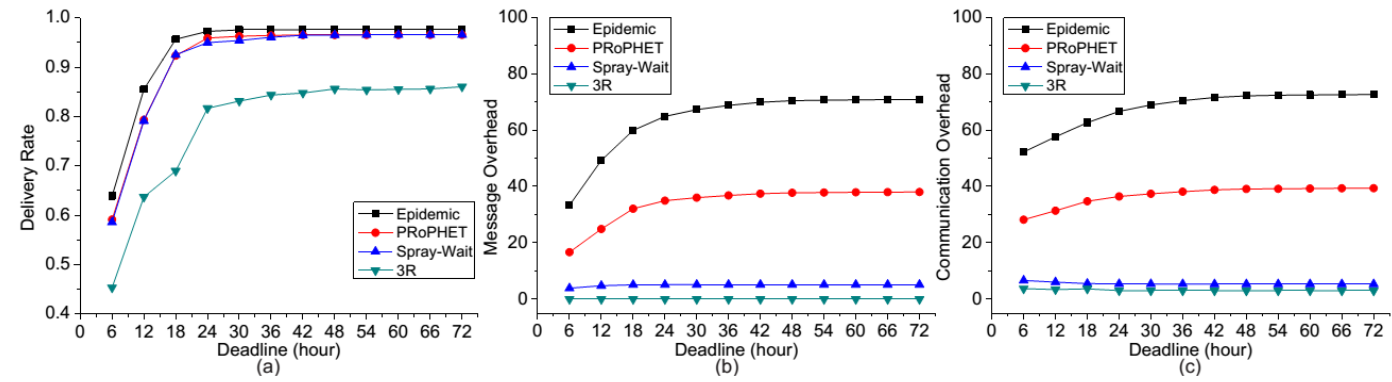

Fig. 6. Performance evaluation with different packet lifetimes using INFOCOM trace, (a) delivery rate, (b) message overhead, (c) communication overhead.

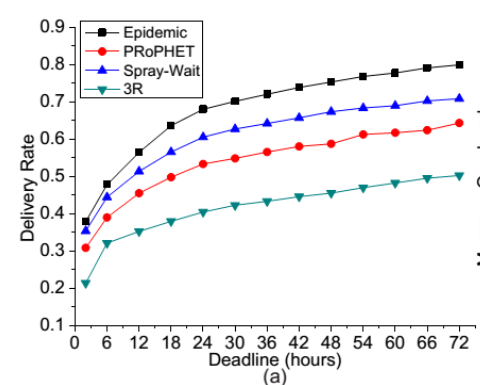

(a)

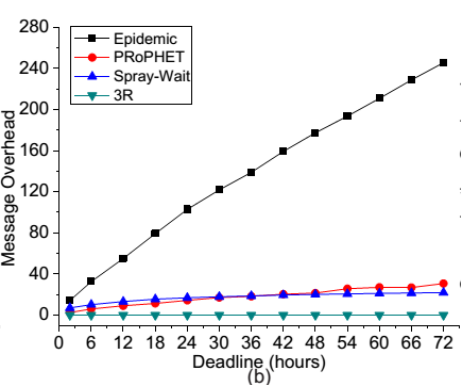

(b) (hours)

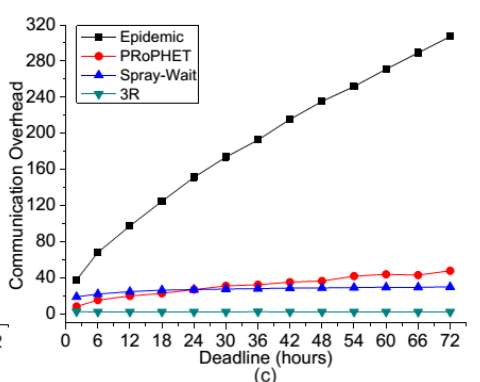

(c)

Fig. 7. Performance evaluation with different packet lifetimes using MIT Reality trace, (a) delivery rate, (b) message overhead, (c) communication overhead. 
(2) MIT Reality Trace: The simulation results with MIT Reality trace are similar as shown in Fig. 7. It can be seen in Fig.7 (a) that the delivery rate for all the routing protocols keep growing as the packet lifetime increases. It is because MIT Reality trace lasts for 1 month and it is longtime dependent, thus a longer packet lifetime always provides a larger probability of successful delivery. In this simulation, as shown in Fig.7 (b) and (c) the overheads of Epidemic increase dramatically both due to longer packet lifetime and larger device population. Whereas, the overheads variations for other routing protocols are similar to the results using INFOCOM data trace.

This set of simulations demonstrate that a longer packet lifetime can provide a higher delivery rate for DTNs, since there is more chance to encounter the destination node either by direct contact or multi-hop message forwarding. The overheads of flooding-based routings increase significantly, while the prediction-based routings are more efficient, especially when the DTNs have long-time dependent contact patterns.

\section{SECURITY EXPERIMENTS}

\subsection{Black Hole Attack and Attack model}

We also investigate the security issue inside DTN protocols. Due to the simplicity of routing design, the protocols we evaluated appear with bare capability to handle hostile attacks from the unstable DTN environment. To test their security strengths, we perform black hole attack which interrupt message spread in the network. Similar to the black hole in universe, the black hole nodes in DTN assimilate all incoming messages and never forward them to others. In the simulation, we randomly select a fixed amount of attacker nodes from the entire population. The amount of attacker nodes is decided by a coefficient called attack opportunity, which measures the badness of DTN environment. The attacker nodes do not originate any message, and all packets will be destroyed after received by these attacker nodes. To make a fair comparison for history-based protocols, the attacker nodes still store and update their contact history, thus sometimes the intruders might be treated as a good relay for some messages. To remove the impact of further losing packets such as message discard from memory overflow, all of the nodes in the simulation have infinite buffer size.

\subsection{Security Test with Black Hole Attack}

(1)INFOCOM: Fig. 8 evaluates the performance of protocols with a steadily increased attack opportunity using the INFOCOM trace. From Fig. 8 (a), we can see that all of the protocols have very similar decrease trend when attack opportunity grows, but $3 \mathrm{R}$ performs much worse than others. Due to the nature of single-message-forward scheme, the lost messages are always irretrievable after they are consumed by attacker nodes. This brings a huge performance degrade for $3 \mathrm{R}$. The rest of the protocols gain a higher and very similar performance. This is because they are all replication-based schemes. Destroying a message replica by an attacker node is not a critical problem for them since the destroyed message might have more copies exist in somewhere else in the network. In (b) and (c), Epidemic still has the highest overheads because of its blind flooding. There is a very interesting point is $3 \mathrm{R}$ and Spray and Wait which have obvious increase on communication overhead at the very late stage. The reason of this for $3 \mathrm{R}$ is that nodes forward original messages through multiple hops and most of them eventually meet attackers, but unfortunately the transmission history of the destroyed messages still have to be counted into the overheads. This result in $3 \mathrm{R}$ has a very poor delivery rate but still gains a great grow on communication overhead. The reason for Spray and Wait is that it propagates message 
copies as a binary tree. The attacker nodes might receive a large number of copies for a single message (e.g. a attacker nodes could receive $2 / n$ of copies at most when it directly receives them from the source node) and then destroy all of them. Hence, Spray and Wait is very sensitive to the amount of message copies. As the attack opportunity grows, the number of successfully delivered message drops faster than the number of message copies it generated. Hence, the CO of Spray and Wait is proportional to attack opportunity.

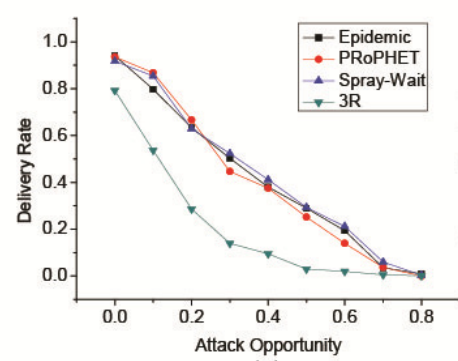

(a)

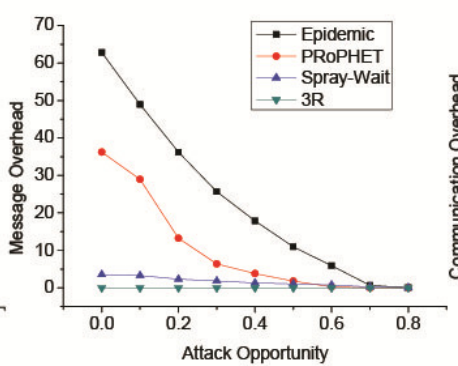

(b)

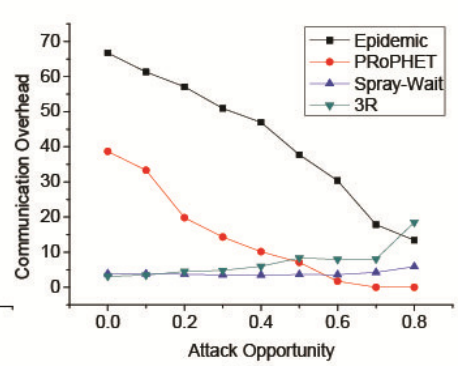

(c)

Fig. 8. Performance evaluation with different black hole attack opportunity using INFOCOM trace, (a) delivery rate, (b) message overhead, (c) communication overhead.

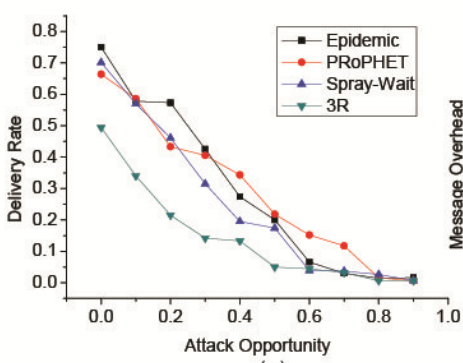

(a)

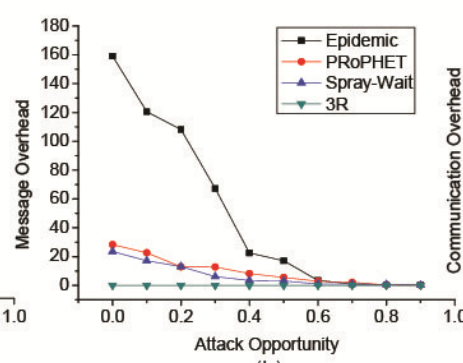

(b)

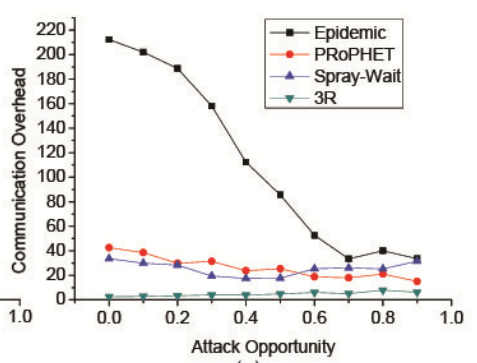

(c)

Fig. 9. Performance evaluation with different black hole attack opportunity using MIT Reality trace, (a) delivery rate, (b) message overhead, (c) communication overhead.

(2)MIT Reality: Fig. 9 shows the result using the MIT Reality trace. It can be seen that the performance differences between protocols appear much more obvious than that in the INFOCOM trace. This is because MIT trace contains larger amount of noise and inactive nodes. In Fig. 9 (a), 3R still has an unexpected performance as above. PRoPHET performs slightly worse than Epidemic and Spray and Wait when the attack opportunity is less than 0.4, but overtakes them afterward. It is because PRoPHET takes the advantage of accurate routing prediction compared to flooding-based protocols. Epidemic has a little bit higher delivery rate than Spray and wait with a incredible high attack opportunity because Epidemic do not have message replication limit. In Fig. 8 (b) and (c), it can be seen that Epidemic still has very high overheads. Different from previous experiments, PRoPHET and Spray and wait have very close overheads. This is because the node population in MIT is much larger than that in INFOCOM, and Spray and Wait needs more message copies to achieve a relative high delivery rate.

In this experiments, it can be seen that single-message-forward protocol is not a good solution under a vulnerable environment. Although all of them did not intentionally provide any solution for security issue, replication-based protocol performs slightly better in a highly stressed 
environment. The overheads of prediction-based protocol are also acceptable, which indicates it is able to handle the restricted hardware situation in DTNs.

\section{CONCLuSion}

Due to the property of Delay Tolerant Networks for no guaranteed continuous connectivity, choosing a proper routing protocol and finding its optimal configuration are challenging problems. In this paper, we evaluate some existing representative routing protocols for DTNs through extensive trace-based simulations. Flooding-based routing protocols, such as Epidemic and Spray and Wait, can achieve better delivery rate by sacrificing the memory, while predictionbased routings, like PRoPHET and 3R, are more efficient when considering the delivery overheads. Although all of the protocols we evaluate have rare capability to handle the hostile attack from adversary users, the prediction-based is able to achieve a relative higher delivery rate. This paper provides important guidance for routing protocols design. Our future work is to design an efficient routing protocol for DTNs with high delivery rate and low delivery overheads.

\section{REFERENCES}

[1] T. Cao, X. Zhang, L. Kong, X. Liu, W. Shu, and M. Wu, "Traffic aware routing in urban vehicular networks," in WCNC, pp. 2004-2009, 2013.

[2] P. Juang, H. Oki, Y. Wang, M. Martonosi, L. S. Peh, and D. Rubenstein, "Energy-efficient computing for wildlife tracking: Design tradeoffs and early experiences with zebranet," SIGARCH Comput. Archit. News, vol. 30, no. 5, pp. 96-107, 2002.

[3] Y. Zhang and J. Zhao, "Social network analysis on data diffusion in delay tolerant networks," in MobiHoc, pp. 345-346, 2009.

[4] A. Vahdat and D. Becker, "Epidemic routing for partially-connected ad hoc networks," tech. rep., 2000.

[5] T. Spyropoulos, K. Psounis, and C. S. Raghavendra, "Spray and wait: An efficient routing scheme for intermittently connected mobile networks," in WDTN, pp. 252-259, 2005.

[6] A. Lindgren, A. Doria, and O. Schel'en, "Probabilistic routing in intermittently connected networks," SIGMOBILE Mob. Comput. Commun. Rev., vol. 7, pp. 19-20, July 2003.

[7] J. Burgess, B. Gallagher, D. Jensen, and B. Levine, "Maxprop: Routing for vehicle-based disruptiontolerant networks," in INFOCOM, pp. 1-11, April 2006.

[8] Q. Yuan, I. Cardei, and J. Wu, "An efficient prediction-based routing in disruption-tolerant networks," IEEE Trans. Parallel Distrib. Syst, vol. 23, no. 1, pp. 19-31, 2012.

[9] L. Vu, Q. Do, and K. Nahrstedt, “3r: Fine-grained encounter-based routing in delay tolerant networks," in WoWMoM, pp. 1-6, 2011.

[10] A. Lindgren, A. Doria, E. Davies, and S. Grasic, "Probabilistic routing protocol for intermittently connected networks," Internet-Draft, 2012.

[11] J. Scott, R. Gass, J. Crowcroft, P. Hui, C. Diot, and A. Chaintreau, "CRAWDAD data set cambridge/haggle (v. 2006-01-31).” http://crawdad.org/cambridge/haggle/, 2006.

[12] N. Eagle and A. (Sandy) Pentland, "Reality mining: sensing complex social systems," Pers. Ubiq. Comput., vol. 10, no. 4, pp. 255-268, 2006.

[13] D. Kotz, T. Henderson, and I. Abyzov, "CRAWDAD data set dartmouth/campus (v. 2004-12-18)." http://crawdad.org/dartmouth/campus, 2004.

[14] J. Shen, S. Moh, and I. Chung, "Routing protocols in delay tolerant networks: A comparative survey," in Proceeding of 23rd International Technical Conference on Circuits/systems, Computer and communications, pp. 1577-1580, 2008 


\section{Authors}

Luming Wan, is a Master candidate from Department of Computer Science, University of Otago, New Zealand. He obtained B.S. degree from University of Otago, New Zealand in 2012. His research interests include Delay Tolerant Network (DTN), Social Network, etc.

Feiyang Liu, is a PhD candidate from Department of Computer Science, University of Otago, New Zealand. He obtained B.S. and M.S. degrees of telecommunication engineering from Xidian University, China in 2009 and 2012, respectively. His research interests include Network on Chip (NoC), Optical Network on Chip (ONoC), Delay Tolerant Networks (DTN), etc.

Yawen Chen, Yawen Chen obtained her PhD degree in Computer Science from The University of Adelaide in Australia in 2008. After her PhD study, she worked as postdoctoral researcher at Royal Institute of Technology (KTH) in 2009. She has been a Lecturer in the University of Otago in New Zealand since 2011. Her research interests include resource optimization and performance evaluation in computer networking and computer architecture (optical networks, interconnection networks, green computing, and etc).

Haibo Zhang, received the MSc degree in Computer Science from Shandong Normal University, China in 2005, and the PhD degree in Computer Science from the University of Adelaide, Australia in 2009. From 2009 to 2010, he was a postdoctoral research associate at Automatic Control Laboratory, KTH, Sweden. Currently he is a lecturer at Computer Science department of University of Otago, New Zealand. His research interests include real-time industrial wireless communications, wireless sensor/ad hoc networks, delay-tolerant networks, green computing, distributed algorithms and protocol design. 